\title{
Atomic force microscopy study of the antibacterial effect of fosfomycin on methicillin-resistant Staphylococcus pseudintermedius
}

\author{
Suresh Neethirajan • Matthew DiCicco
}

Received: 15 April 2013/ Accepted: 16 July 2013/Published online: 31 July 2013

(C) The Author(s) 2013. This article is published with open access at Springerlink.com

\begin{abstract}
The influence of fosfomycin on methicillinresistant Staphylococcus pseudintermedius (MRSP) as the target cell was studied by atomic force microscopy (AFM). Nanoscale images of the effects of the antibiotic fosfomycin on this gram-positive bacterium's cell were obtained in situ without fixing agents. Our study has demonstrated substantial morphological and topographical differences between the control and fosfomycin-treated MRSP cells. The AFM investigations further revealed the rough surface morphology and a $30 \%$ shrinkage in size of the fosfomycin-treated cell and the leakage of cytoplasmic components from the cell. The damage of cell membrane integrity and the cell surface degradation as observed elaborates the antibacterial activity of fosfomycin. The AFM image analysis also reveals that the fosfomycin inhibits cell division, and prevents the adhesion on the surface discouraging the biofilm attachment. The microtitre plate assay results conform to the atomic force microscopy image analysis. This is the first visual demonstration of the effect of fosfomycin on MRSP cells.
\end{abstract}

Keywords Staphylococcus pseudintermedius - Atomic force microscopy - Fosfomycin - Cell wall antimicrobial . Lipopolysaccharide

\section{Introduction}

Staphylococcus pseudintermedius is a major cause of morbidity and has been increasingly found in the skins and

S. Neethirajan $(\varangle) \cdot$ M. DiCicco

BioNano Laboratory, School of Engineering, University

of Guelph, Guelph N1G 2W1, Canada

e-mail: s.neethi@uoguelph.ca ears of companion animals leading to economic loss in animal health. Methicillin-resistant Staphylococcus pseudintermedius (MRSPs) are the frequent cause of pyoderma, urinary tract infections and otitis (Borjesson et al. 2012; Kadlec et al. 2010; Rubin et al. 2011). As an opportunistic pathogen, these gram-positive Staphylococcus pseudintermedius cocci can occasionally cause infections in humans (Chuang et al. 2010; Hoovels et al. 2006). Due to the emergence and widespread dissemination of MRSP, it becomes an expensive and a serious challenge to control or eradicate these pathogenic biofilms using antibiotics. MRSP ST71 has been reported (Stegmann et al. 2010) to cause human infection from companion animals demonstrating the therapeutic challenge and its zoonotic potential.

MRSP isolates are resistant to several clinically useful antibiotics including $\beta$-lactam agent such as clarithromycin (DiCicco et al. 2012). Fosfomycin is a wide spectrum antibiotic used for treating urinary tract and genital tract infections caused by both gram-positive and gram-negative bacterial strains (Falagas et al. 2010). Fosfomycin exert antimicrobial activity by inhibiting UDP-N-acetylglucosamine enolpyruvyl transferase enzyme (Eschenburg et al. 2005) that catalysis the first step in bacterial cell-wall synthesis within the cell. Fosfomycin has the ability to penetrate into the fluid of the interstitial space of soft tissues (Frossard et al. 2000) to reach levels sufficient to substantially inhibit the growth of relevant bacteria at the target site. The pharmacokinetic and pharmacodynamic properties of fosfomycin facilitate design of drug doses with clinically relevant concentrations for sites such as lungs, bone, soft tissue, and serum and heart valves (Roussos et al. 2009). So far, there have been no reports of fosfomycin's cross-resistance to other antibiotics. Further, the lower molecular weight of fosfomycin $(138.059 \mathrm{~g} / \mathrm{mol})$ makes it an attractive agent for designing lower microbial 
inhibition concentration drugs. Hence, fosfomycin could be a promising alternate candidate for the treatment of infections caused by MRSP biofilms.

To further extend the understanding of clinical relevance in recommending fosfomycin for treating MRSP biofilms, it is necessary to understand the antimicrobial activity of fosfomycin at the nanoscale level. Atomic Force Microscopy (AFM) imaging technique overcomes the drawbacks of Scanning Electron Microscopy (SEM) such as the need for conductive coating on biofilm samples for SEM imaging, extensive sample preparation techniques, and the vacuum requirement during imaging. Confocal Laser Scanning Imaging (CLSM) can image the bacterial cells at their physiological conditions, but offers only at macroscopic level. Hence, AFM proves to be superior compared to both SEM and CLSM in imaging the biofilms at the nanoscale level. AFM experiments has been carried out to investigate the effects of antimicrobial and antibiotic properties of chitosans (Eaton et al. 2008), kyotorphin peptide (Ribeiro et al. 2012), carvacrol (Storia et al. 2011) on Staphylococcal aureus biofilms. The nanoscale visualization and characterization of Staphylococcus aureus cells (Ribeiro et al. 2012) demonstrated that kyotorphin perturbed the bacterial cell-wall membrane by blebbing, lysis and disruption. The molecular mechanism of nanoconjugated vancomycin (Chakraborty et al. 2012) towards antimicrobial therapeutic approaches in treating Staphylococcus aureus at the nanoscale proved that AFM is a potential tool in understanding the types of antimicrobial induced bacterial cell-wall damage.

The effects of various antibiotic candidates on MRSA have been studied in detail; however to-date there has been no nanoscale investigation of antimicrobial agents on MRSPs. Both MRSPs and MRSAs are gram-positive cocci, but MRSPs are distinctly different than MRSAs. The spread of mecA gene in S. aureus and S. pseudintermedius occur via independent routes, and hence MRSAs are distinctly different than the MRSP from an evolutionary point of view (Sasaki et al. 2007). Moreover, the resistance pattern to antibiotics is different between MRSA and MRSP due to their different biological behaviors. Here, we evaluate the evidence on fosfomycin as a treatment option against MRSP biofilms using atomic force microscopy. The objective of this work is to elucidate the antimicrobial activity of fosfomycin on MRSP using AFM imaging techniques. This is the first report on the visual investigation of the effect of fosfomycin on MRSPs.

\section{Methods}

Chemicals and materials

MRSP A12 strains collected from the Centre for Public Health and Zoonotic Diseases of the Ontario Veterinary
College was used to investigate the effect of fosfomycin. Fosfomycin (FOS) was acquired from Sigma Chemical Co (St. Louis, MO, USA).

Preparation of bacterial samples for morphology studies

MRSP A12 isolate was sub-cultured for $24 \mathrm{~h}$ at $35^{\circ} \mathrm{C}$ in Columbia Agar with $5 \%$ sheep blood plates. Upon $24 \mathrm{~h}$, the isolate was added to nutrient-rich tryptic soy broth supplemented with $1 \%$ glucose (TSB-G) in $10 \mathrm{ml}$ tubes and was grown to late mid-log phase at $1 \times 10^{6} \mathrm{CFU} / \mathrm{ml}$. The cells in $1.0 \mathrm{ml}$ culture were centrifuged in a Scilogex Model D3024 microfuge at 3,500 rpm for $3 \mathrm{~min}$ at room temperature, and washed 3 times with 1 volume of sterile analytical-grade water. The pellet was again suspended in deionized distilled water and the concentration of the bacteria was measured by a spectrophotometer at $420 \mathrm{~nm}$. Freshly cleaved mica sheets were added to petri-dishes containing the bacterial cell culture suspension with and without $8 \mu \mathrm{g} / \mathrm{ml} \mathrm{FOS}$ and incubated for $24 \mathrm{~h}$ at $35^{\circ} \mathrm{C}$. At $4,8,12$ and $24 \mathrm{~h}$ of incubation, the mica sheets were gently removed using fine tip tweezers, washed in free-flowing nanopure water to remove the freely attached cells and dried at room temperature for $3 \mathrm{~h}$ before imaging. AFM imaging was carried out for both the control samples and the bacterial culture treated with fosfomycin. Analysis was done with duplicate cultures for each time point.

\section{AFM imaging}

MRSP A12 cells were imaged in air with a tapping mode atomic force microscope (Dimension Icon SPM, Bruker) before and after being treated with antibiotics. AFM height, amplitude and phase images were obtained in AC mode on the air-dried mica substrates. A triangular Si cantilever tip (Bruker AFM Probes, Camarilla, CA, USA) with a spring constant of $0.35 \mathrm{~N} / \mathrm{m}$ and a resonance frequency of $18 \mathrm{kHz}$ was used. A scan speed of $0.7-1.5 \mathrm{~Hz}$ was set and resulted in a final resolution of 512 by 512 pixels.

\section{Results and discussion}

The cocci or the spheroid cell shape morphology is evident from the AFM images (Fig. 1) of the untreated cells. AFM image analysis revealed that the surface ultrastructure of both MRSP ST72 strains and the cells treated with fosfomycin (Fig. 2) are completely different. The healthy MRSP cells are distinguishable from the fosfomycin exposed cells. The untreated MRSP cells have a relatively smooth surface without pores or any ruptures in comparison with the grooves on the fosfomycin-treated cells. Grooves or depressions or holes distributed on every cell surface 
Fig. 1 Topographical images of MRSP A12 cells before

(a) and after being treated with fosfomycin (b)
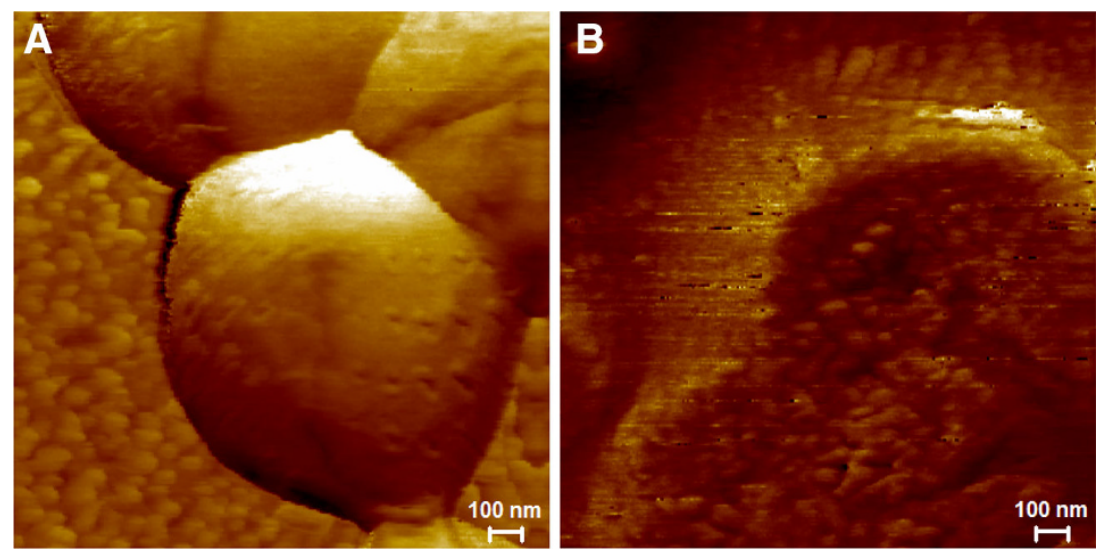
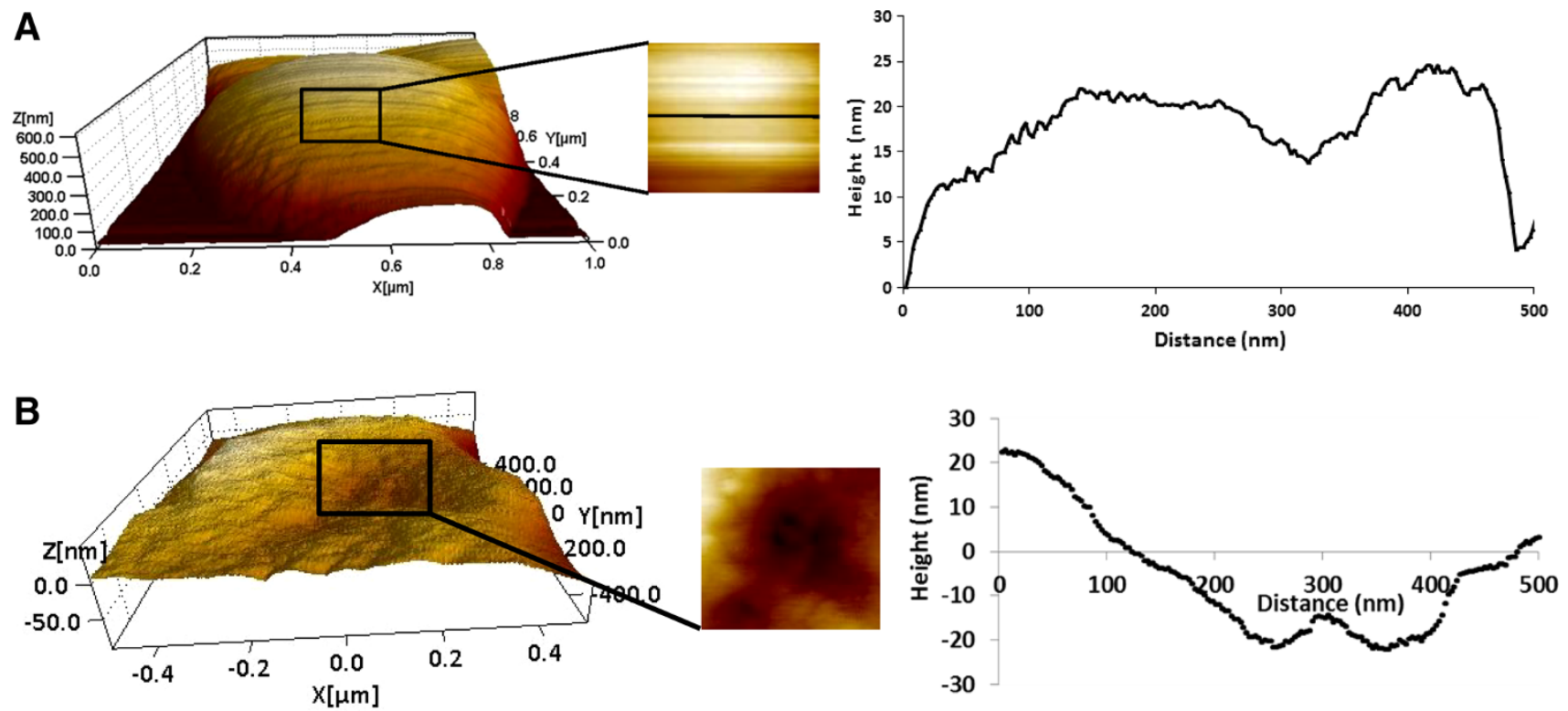

Fig. 2 AFM images of MRSP A12 control (a) and fosfomycin-treated (b) cells. Shown are fixed whole cells (a, b) and corresponding surface ultrastructure (c, d). Bars $100 \mathrm{~nm}$

demonstrate the shape alterations induced by fosfomycin. The surface of fosfomycin-treated MRSP A12 biofilms (Fig. 2b) has densely packed surface subunits made of small aggregates of surface molecules.

Significant differences in cellular dimensions between control and the fosfomycin-treated MRSP cells (Fig. 3a, b) are observed from the AFM images. The statistical results of the average diameter of the cocci-shaped grampositive MRSP A12 untreated cells and the fosfomycintreated cells obtained from the AFM height images are $1,008 \pm 123$ and $780 \pm 100 \mu \mathrm{m}$ respectively. The pharmacological antimicrobial mechanism of fosfomycin is based on the cell-wall biosynthesis inhibition process. Briefly, fosfomycin inactivates the MurA proteins which are present at the transition between cytoplasmic synthesis of the uridine diphosphate (UDP)-linked precursor by mimicking phosphoenolpyruvate and by irreversibly modifying the critical active-site nucleophile Cys115 through a covalent linkage (Lovering et al. 2012; Kahan et al. 1974). The structure of fosfomycin is shown in Fig. 4. A therapeutic dosage of $8 \mu \mathrm{g} / \mathrm{ml}$ concentration of fosfomycin was chosen to test the efficacy against MRSP biofilms based on the Clinical and Laboratory Standards Institute guidelines (CLSI, PA, USA) and this dosage can be attained in humans by oral or parenteral administration. Hence the influence of different concentrations of fosfomycin on MRSP A12 cells was not explored in this study.

The surface roughness of the cells was calculated as a quantitative assessment of the surface texture with the average of the vertical deviations from the nominal surface over a specified area (Dong et al. 2011). The roughness differences on the ultra-structural surface of fosfomycintreated cells have accounted for the change in cell shape 

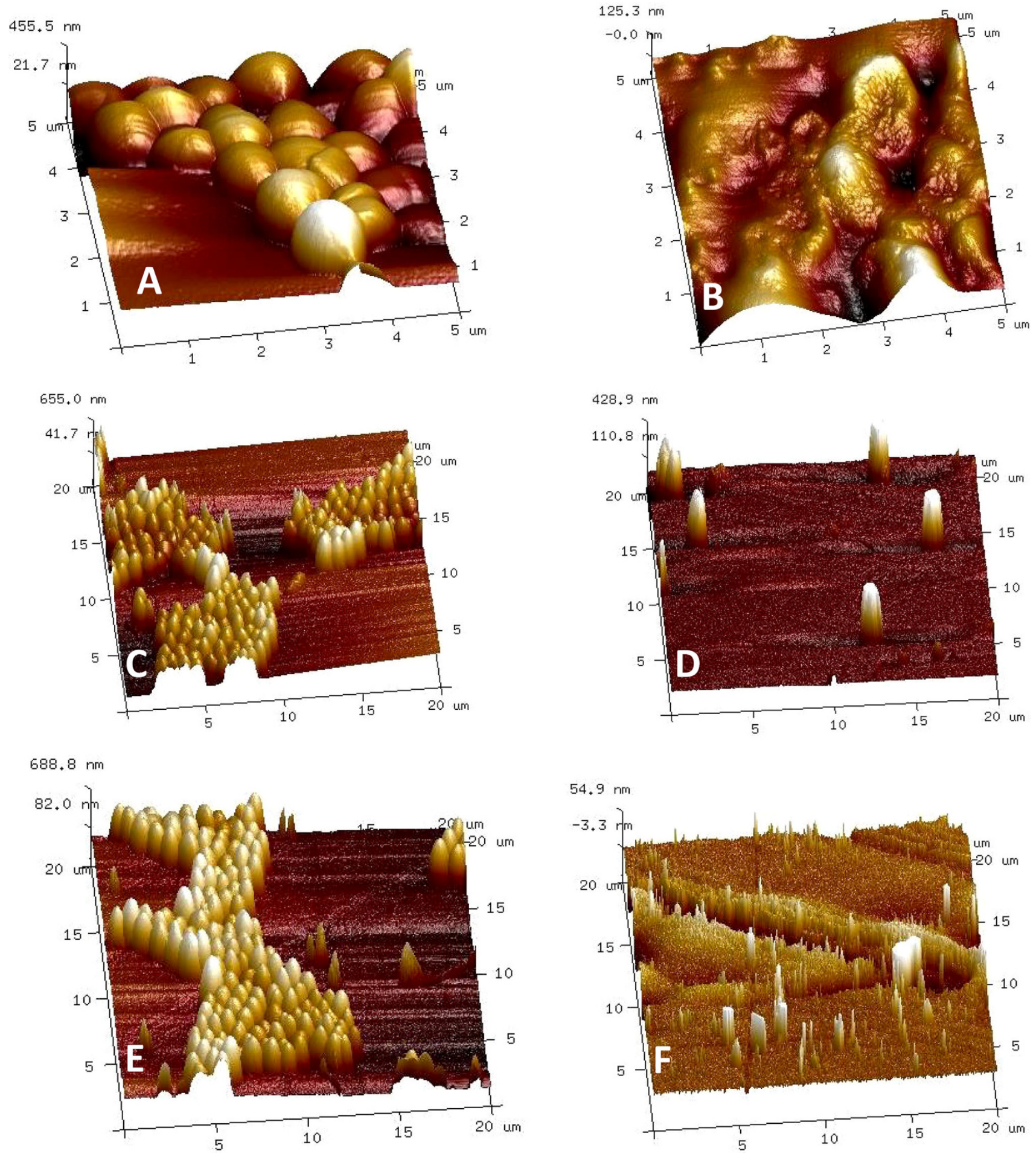

Fig. 3 Time dependence of fosfomycin effects on MRSP imaged by AFM. Three-dimensional orthogonal projection images (derived from the height data) of untreated MRSP cells (a, c, e), and MRSP cells treated with $8 \mu \mathrm{g} / \mathrm{ml}$ fosfomycin (b, d, f). Images were acquired

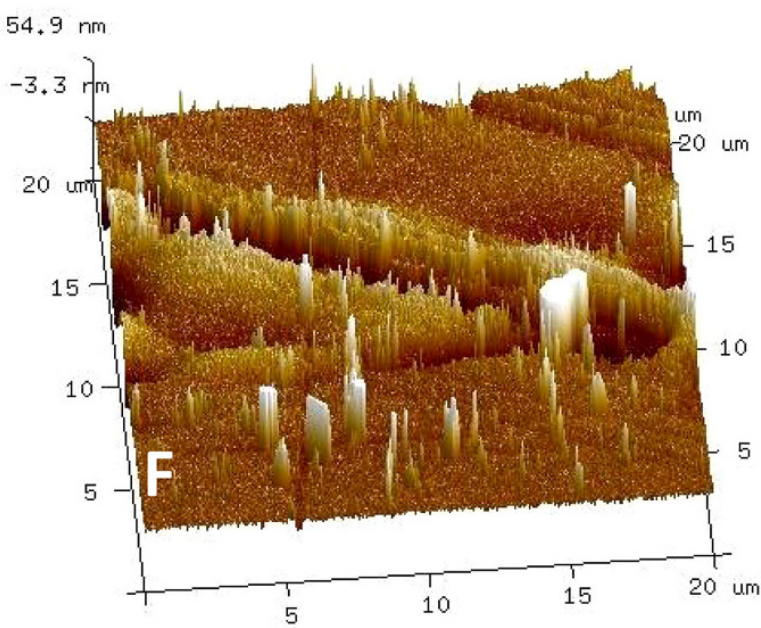

following the treatment of the MRSP cells for $4 \mathrm{~h}(\mathbf{a}, \mathbf{b}), 8 \mathrm{~h}$ (c), d) and $12 \mathrm{~h}(\mathbf{e}, \mathbf{f})$. Total scanning area for the 8- and 12-h treated MRSP A12 images are $20 \times 20 \mu \mathrm{m}^{2}$

The action effects of fosfomycin on the MRSP cells over greatly. The increase in the numerical surface roughness suggests the efficiency of fosfomycin in interacting with the cellular membrane. various periods of treatment are shown in Fig. 3. Timecourse study investigating the effect of fosfomycin on the 


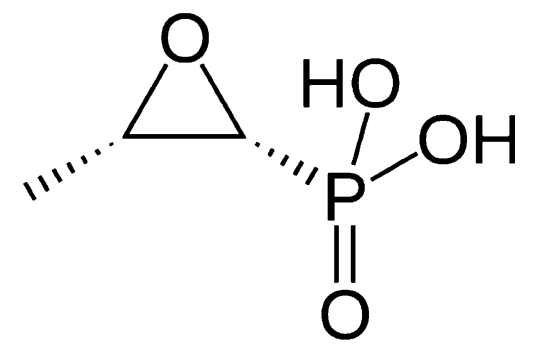

Fig. 4 Structure of fosfomycin

MRSP bacterial surface provides better understanding of antibacterial mechanism. The nature and degree of outer membrane damage on the MRSP cells caused by fosfomycin treatment over 4,8 and $12 \mathrm{~h}$ inducing substantial topographical changes such as the $30 \%$ shrinkage in the cell size and reduced surface coverage can be observed. Increased incubation time and the increased fosfomycin concentration are correlated well with the bacterial outer membrane damage as observed by the decreased height from the Fig. 3b, d, f.

On control samples, the MRSP cells are adhered on the mica surface in well-organized patterns with maximum contact maintaining structural integrity. In comparison, the fosfomycin-treated cells appear to lift-off and maintain physical separation. Clustering effect is observed on control samples as the cells clump together to form biofilm. AFM image analysis of fosfomycin-treated MRSP biofilms shows that the cells are in smaller groups and are attached individually and sparsely distributed across the mica substrate surface. The total numbers of cells present on the treated mica sheets were substantially reduced by $80 \%$ and the remaining cells displayed a tendency to scatter. In addition, the fosfomycin-treated cells have collapsed and flattened indicating the characteristic damages such as the reduced height and the diameter. The structural alterations characterized by the loss of regular shape are attributed to the possible cell-wall biosynthesis inhibition mechanism of fosfomycin. The cell-wall degradation is more pronounced in 8-h treated cells compared to the 4-h treated MRSP cells.

The occurrence of the defects in the cell wall and the protoplasts surrounding the cells (Fig. 5a) indicates the action of fosfomycin on the bacterial peptidoglycan layer. The cellular proteins have leaked from the cells and appear as globular structure enclosing the cell clusters. The surface roughness on the fosfomycin-treated cells indicates the presence of macro pore distribution (Fig. 5b).

The gram-positive cell is dense with about $20-80 \mathrm{~nm}$ thickness (Dmitriev et al. 2004) and consists of numerous interconnecting layers of peptidoglycan. The chemical make-up of this cell wall consists of cables of cross-linked glycan strands of about $50 \mathrm{~nm}$ in width. The cell wall also consists of teichoic acids and lipoteichoic acids (Silhavy et al. 2010; Kohanski et al. 2010), while the outer surface of peptidoglycan is covered with the surface proteins offering security for the cell's survival. Hence, the damage to the cell wall causes loss of inner contents of cell and thereby leading to cell death. The AFM phase images between control and fosfomycin-treated cells shows that the phase difference is large indicating that the cell surfaces had different viscoelasticity of the cell surface regions. This large phase difference also corresponds to the

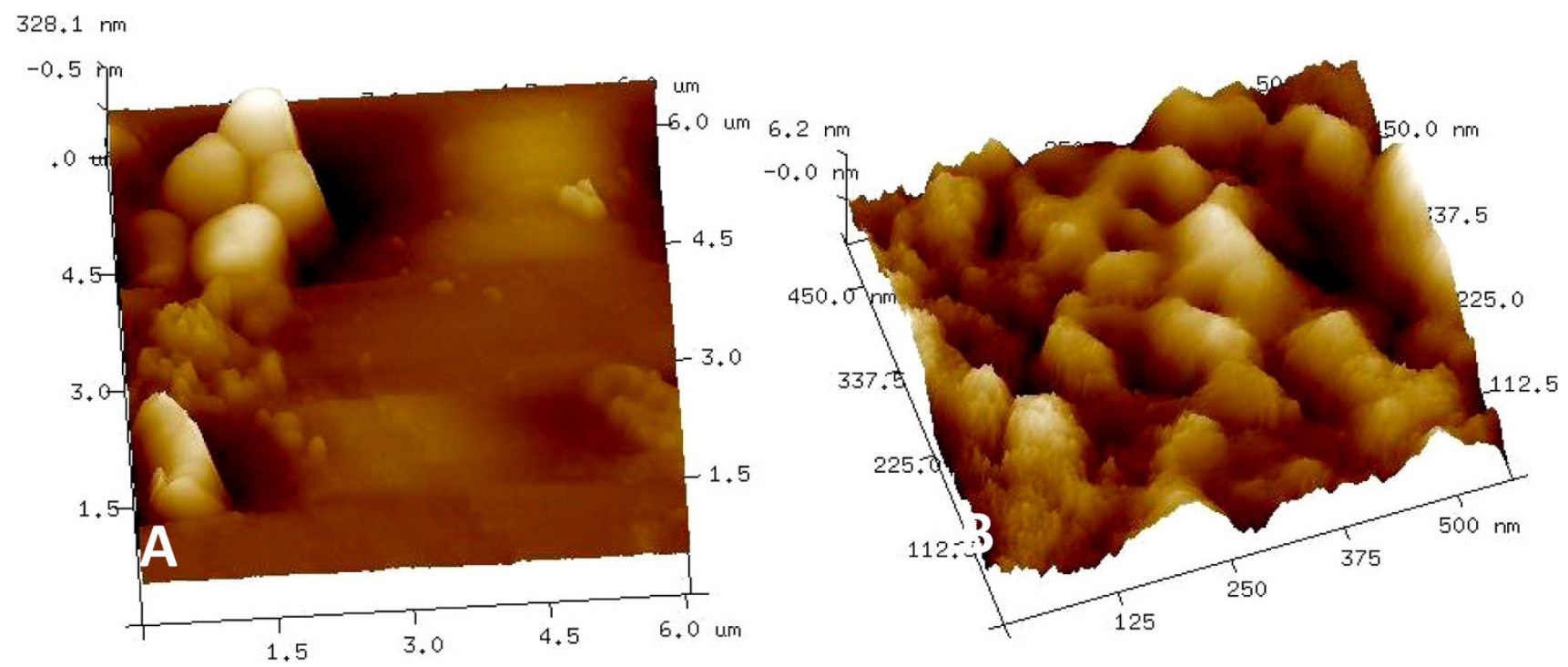

Fig. 5 a Atomic force micrograph showing the leaking of contents from fosfomycin-treated MRSP A12 cells on the mica surface. b AFM image showing the surface ultrastructure roughness with mesopores 


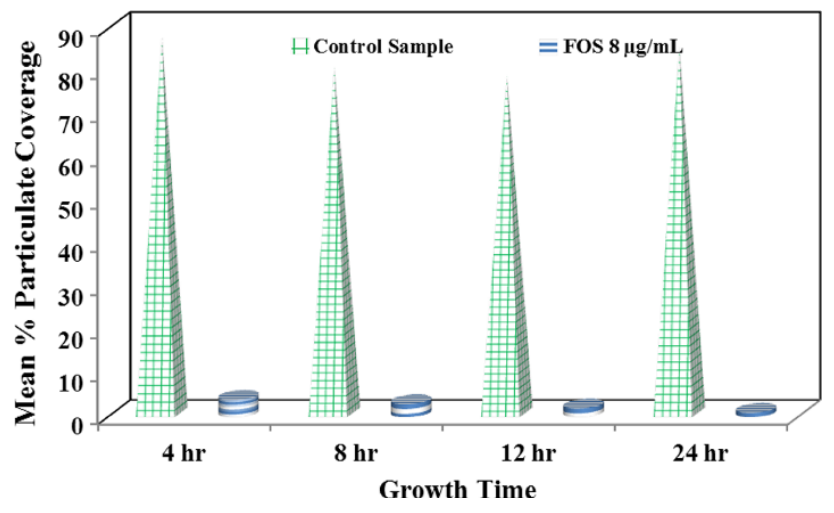

Fig. 6 Percent biofilm coverage on mica sheet surface over 4, 8, 12 and $24 \mathrm{~h}$ time periods as observed by Atomic Force Microscopy. Image analysis of particulate coverage of AFM biofilm images using ImageJ software shows that a significant difference $(P<0.05)$ in surface coverage area exists between fosfomycin-treated and control MRSP samples

height difference showing that the fosfomycin treatment had exposed the cell surface.

Treatments of $8 \mu \mathrm{g} / \mathrm{mL}$ of fosfomycin on MRSP biofilms were found to reduce adherence and biofilm structure on the mica sheet surface. Percent particulate on the mica surfaces decreased significantly $(P<0.05)$ between the fosfomycin-treated samples and the control MRSP A12 biofilm samples observed through image analysis (Fig. 6). Comparisons between the samples indicate that surface area coverage by MRSP biofilm decreased from 85 to $3 \%$ over $24 \mathrm{~h}$. This large difference in surface coverage indicates the potential of fosfomycin in influencing the adhesion kinetics of MRSP on material surfaces.

It should be emphasized that the AFM imaging of the MRSP samples on mica sheet surfaces was conducted under ambient conditions, as it enables high-resolution imaging. Investigations of the MRSP biofilms and its adhesion kinetics and antimicrobial mechanism are currently being undertaken using AFM and nanoscale imaging tools. In view of a growing resistance of bacteria to many antibiotics, it is important that a wider range of materials are examined for their antibacterial potential and the effects of fosfomycin on MRSP biofilms are of interest to microbiologists and of pharmaceutical scientists. Further study is warranted to elucidate the genetic mechanisms leading to the change in cell morphology to understand various the multi-drug resistances of MRSP biofilms.

\section{Conclusion}

The use of atomic force microscopy allowed to characterize the detailed effect of fosfomycin, and thereby helped to evaluate the range of morphological changes of the bacterial cell surface. Fosfomycin induces pore like damages on the envelope of the MRSP cell, and also cell-wall damage leading to leaking of cell's contents. It appears that the fosfomycin has disrupted the peptidoglycan cell-wall layer and has targeted the membrane components. The AFM image analysis also reveals that the fosfomycin inhibits cell division, and prevents the adhesion on the surface discouraging the biofilm attachment. The microtitre plate assay results conform to the atomic force microscopy image analysis. This is the first visual demonstration of the effect of fosfomycin on MRSP cells. The effect of fosfomycin on the morphometric and the surface structure of the MRSP bacterial cells reveal that fosfomycin could be an effective antibiotic agent for treating infections caused by MRSP biofilms. Further research on in vivo studies on the efficacy of fosfomycin in treating MRSP biofilms is recommended.

Acknowledgments The authors sincerely thank Dr. Scott Weese and his team at the Department of Pathobiology, Ontario Veterinary College of the University of Guelph for providing bacterial strains and the insightful discussions. We gratefully acknowledge the support for this work from the Natural Sciences and Engineering Research Council of Canada.

Conflict of interest The authors declare that they have no competing interests.

Open Access This article is distributed under the terms of the Creative Commons Attribution License which permits any use, distribution, and reproduction in any medium, provided the original author(s) and the source are credited.

\section{References}

Borjesson S, Landen A, Bergstrom M, Andersson UG (2012) Methicillin-resistant Staphylococcus pseudintermedius in Sweden. Microb Drug Resist 18:597-603

Chakraborty SP, Sahu SK, Pramanik P, Roy S (2012) In vitro activity of nanoconjugated vancomycin against drug resistant Staphylococcus aureus. Int J Pharm 436:659-676

Chuang CY, Yang YL, Hsueh PR, Lee P (2010) Catheter-related bacteremia caused by Staphylococcus pseudintermedius refractory to antibiotic-lock therapy in a hemophilic child with dog exposure. J Clin Microbiol 48:1497-1498

DiCicco M, Neethirajan S, Singh A, Weese JS (2012) Efficacy of clarithromycin on biofilm formation of methicillin-resistant Staphylococcus pseudintermedius. BMC Vet Res 8:1-7

Dmitriev BA, Toukach FV, Holst O, Rietschel ET, Ehlers S (2004) Tertiary structure of Staphylococcus aureus cell wall murein. J Bacteriol 186:7141-7148

Dong J, Signo KSL, Vanderlinder EM, Yost CK, Dahms TES (2011) Atomic force microscopy of a ctpA mutant in Rhizobium leguminosarum reveals surface defects linking CtpA function to biofilm formation. Microbio 157:3049-3058

Eaton P, Fernandes JC, Pereira E, Pintado ME, Malcata FX (2008) Atomic force microscopy study of the antibacterial effects of chitosans on Escherichia coli and Staphylococcus aureus. Ultramicroscopy 108:1128-1134 
Eschenburg S, Priestman M, Schonbrunn E (2005) Evidence that the fosfomycin target Cys115 in UDP-N-acetylglucosamine enolpyruvyl transferase (MurA) is essential for product release. J Biol Chem 280:3757-3763

Falagas ME, Kastoris AC, Kapaskelis AM, Karageorgopoulos DE (2010) Fosfomycin for the treatment of multidrug-resistant, including extended-spectrum $\beta$-lactamase producing, Enterobacteriaceae infection: a systematic review. Lancet Infect Dis 10:43-50

Frossard M, Joukhadar C, Erovic BM, Dittrich P, Mrass PE, Houte MV, Burgmann H, Georgopoulos A, Muller M (2000) Distribution and antimicrobial activity of fosfomycin in the interstitial fluid of human soft tissues. Antimicrob Agents Chemother 44: $2728-2732$

Hoovels VL, Vankeerberghen A, Boel A, Vaerenbergh KV, Beenhouwer HD (2006) First case of Staphylococcus pseudintermedius infection in a human. J Clin Microbiol 44:4609-4612

Kadlec K, Schwarz S, Perreten V, Andersson UG, Finn M, Greko C, Moodley A, Kania SA, Frank LA, Bemis DA, Franco A, Lurescia M, Battisti A, Duim B, Wagenaar JA, Duijkeren EV, Weese JS, Fitzgerald JF, Rossano A, Guardabassi L (2010) Molecular analysis of methicillin-resistant Staphylococcus pseudintermedius of feline origin from different European Countries and North America. J Antimicrob Chemother 65: $1826-1828$

Kahan FM, Kahan JS, Cassidy PJ, Kropp H (1974) The mechanism of action of fosfomycin (phosphonomycin). Ann N Y Acad Sci 235:364-386

Kohanski MA, Dwyer DJ, Collins JJ (2010) How antibiotics kill bacteria: from targets to networks. Nat Rev Microbiol 8(6): 423-435
Lovering AL, Safadi SS, Strynadka NCJ (2012) Structural perspective of peptidoglycan biosynthesis and assembly. Annu Rev Biochem 81:451-478

Ribeiro MMB, Franquelim HG, Torcato IM, Ramu VG, Heras M, Bardaji ER, Castanho MARB (2012) Antimicrobial properties of analgesic kyotorphin peptides unraveled through atomic force microscopy. Biochem Biophys Res Commun 420:670-676

Roussos N, Karageorgopoulos DE, Samonis G, Galagas ME (2009) Clinical significance of the pharmacokinetic and pharmacodynamic characteristics of fosfomycin for the treatment of patients with systemic infection. Int J Antimicrob Ag 34:506-515

Rubin JE, Ball KR, Chirino-Trejo M (2011) Antimicrobial susceptibility of Staphylococcus aureus and Staphylococcus pseudintermedius isolated from various animals. Can Vet J 52:153-157

Sasaki T, Kikuchi K, Tanaka Y, Takahashi N, Kamata S, Hiramatsu K (2007) Methicillin-resistant Staphylococcus pseudintermedius in a Veterinary Teaching Hospital. J Clin Microbiol 45:1118-1125

Silhavy TJ, Kahne D, Walker S (2010) The bacterial cell envelope. Cold Spring Harb Perspect Biol 5:1-16

Stegmann R, Burnens A, Maranta CA, Perreten V (2010) Human infection associated with methicillin-resistant Staphylococcus pseudintermedius ST71. J Antimicrob Chemother 65:2047-2048

Storia AL, Ercolini D, Marinello F, Pasqua RD, Villani F, Mauriello G (2011) Atomic force microscopy analysis shows surface structure changes in carvacrol-treated bacterial cells. Res Microbiol 162:164-172 